\title{
Visual attention during pediatric resuscitation with feedback devices: a randomized simulation study
}

\author{
Michael Wagner $\mathbb{D}^{1 凶}$, Peter Gröpel ${ }^{2}$, Felix Eibensteiner ${ }^{1}$, Lisa Kessler ${ }^{1}$, Katharina Bibl ${ }^{1}$, Isabel T. Gross ${ }^{3}$, Angelika Berger ${ }^{1}$ and \\ Francesco S. Cardona (iD) ${ }^{1}$ \\ (c) The Author(s) 2021
}

BACKGROUND: The aim of this study was to investigate the effect of feedback devices on visual attention and the quality of pediatric resuscitation.

METHODS: This was a randomized cross-over simulation study at the Medical University of Vienna. Participants were students and neonatal providers performing four resuscitation scenarios with the support of feedback devices randomized. The primary outcome was the quality of resuscitation. Secondary outcomes were total dwell time (=total duration of visit time) on areas of interest and the workload of participants.

RESULTS: Forty participants were analyzed. Overall, chest compression $(P<0.001)$ and ventilation quality were significantly better $(P=0.002)$ when using a feedback device. Dwell time on the feedback device was $40.1 \%$ in the ventilation feedback condition and $48.7 \%$ in the chest compression feedback condition. In both conditions, participants significantly reduced attention from the infant's chest and mask (72.9 vs. $32.6 \%$ and 21.9 vs. $12.7 \%$ ). Participants' subjective workload increased by $3.5 \%(P=0.018)$ and $8 \%$ $(P<0.001)$ when provided with feedback during a 3-min chest compression and ventilation scenario, respectively.

CONCLUSIONS: The quality of pediatric resuscitation significantly improved when using real-time feedback. However, attention shifted from the manikin and other equipment to the feedback device and subjective workload increased, respectively.

Pediatric Research (2022) 91:1762-1768; https://doi.org/10.1038/s41390-021-01653-w

\section{IMPACT:}

- Cardiopulmonary resuscitation with feedback devices results in a higher quality of resuscitation and has the potential to lead to a better outcome for patients.

- Feedback devices consume attention from resuscitation providers.

- Feedback devices were associated with a shift of visual attention to the feedback devices and an increased workload of participants.

- Increased workload for providers and benefits for resuscitation quality need to be balanced for the best effect.

\section{INTRODUCTION}

Around $2 \%$ of pediatric intensive care unit admissions will experience cardiac arrest. ${ }^{1}$ Successful high-quality cardiopulmonary resuscitation (CPR) of in-house arrest may lead to the discharge of up to half of these patients. ${ }^{1-3}$ Training in a simulation setting improves the quality of CPR. ${ }^{4,5}$ Focus is on adequate chest compressions (CCs) (depth, frequency, recoil) and ventilations (frequency, tidal volume, leak, pressure) as well as few interruptions (short hands-off time). This may be achieved with real-time feedback devices used during resuscitation, which may assist in achieving a better quality of resuscitation..$^{6-11}$ These devices relay information on compression as well as ventilation quality. ${ }^{12-16}$ Even though feedback devices are increasingly being used in educational and clinical settings, the resuscitation guidelines by the European Resuscitation Council still argue against routine implementation ${ }^{17}$ as evidence is still not clear-cut. Previous studies using the same feedback devices as in our study [QCPR Baby (Laerdal, Stavanger, Norway) for CCs and Neo Training (Monivent AB, Gothenburg, Sweden) for ventilations] have shown an improvement of resuscitation and ventilation performance. ${ }^{6,18,19}$ However, these devices may even be detrimental and lead to higher subjective stress. ${ }^{20}$ This increased mental workload may in turn lead to worse performance. ${ }^{21}$ Eye-tracking technology may allow the assessment of visual attention (VA) of healthcare providers during resuscitation. ${ }^{22,23}$ This may allow quantification of distractions during resuscitation and in turn lead to strategies to overcome these during resuscitation.

We therefore aimed to study the impact of feedback devices on resuscitation quality with eye-tracking to analyze participants' performance when supported with feedback devices.

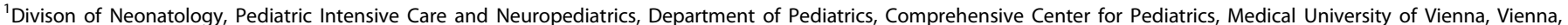

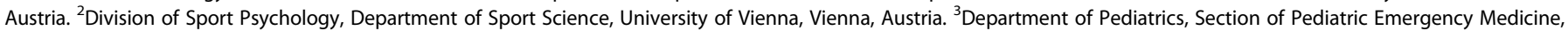
Yale School of Medicine, New Haven, CT, USA. ${ }^{凶}$ email: michael.b.wagner@meduniwien.ac.at

Received: 9 February 2021 Revised: 9 June 2021 Accepted: 30 June 2021

Published online: 21 July 2021 


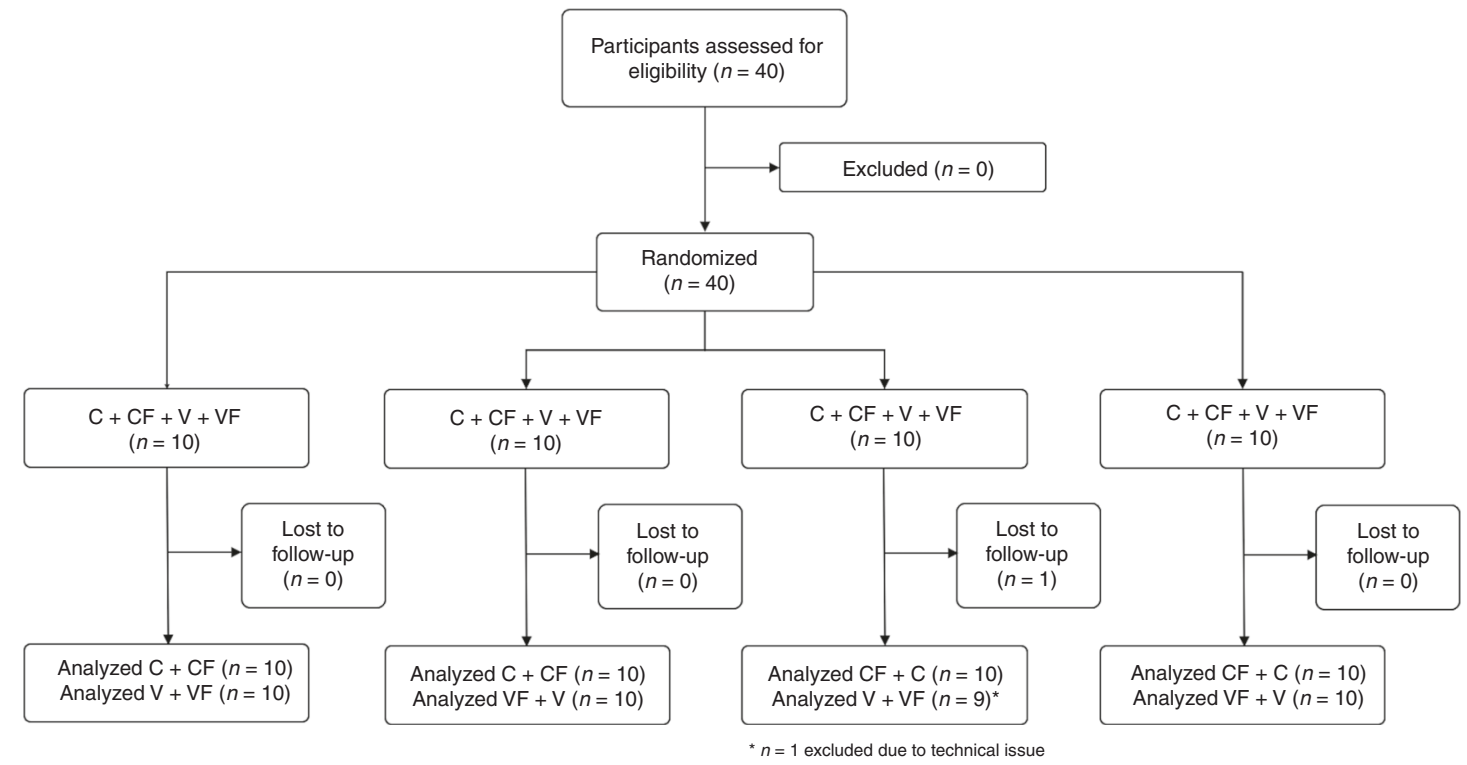

Fig. 1 Flow diagram. $C+C F$ chest compression without/with feedback device, $V+V F$ ventilation without/with feedback device.

\section{METHODS}

This study was a prospective randomized cross-over simulation-based trial conducted at the Medical University of Vienna, Austria. The study protocol was reviewed according to the Consolidated Standards of Reporting Trials (CONSORT). ${ }^{24}$ The ethics committee of the Medical University of Vienna gave this study an exempt status.

\section{Participants}

Medical students in their final year, fellows, nurses, and consultants from our Neonatal Intensive Care Unit were eligible for enrolment.

\section{Study procedure and scenario}

Participants received a brief review of the current pediatric CPR guidelines and an introduction to the feedback devices at the beginning of the study. Participants then signed an informed consent form and completed a questionnaire to assess demographic variables and expertise in pediatric resuscitation. Thereafter, eye-tracking glasses (Tobii 2.0, Tobii AB, Danderyd, Sweden) were calibrated as recommended by the company. Resuscitation teams consisted of the participant and a study nurse. Each participant completed a total of four basic life support scenarios according to the ERC pediatric BLS guidelines (15:2) in a cross-over setting. ${ }^{25}$ Each participant did CC twice, and ventilations (V) twice afterward (see Fig. 1).

\section{Randomization}

The visibility of the feedback device was randomized (sealed envelope) within each pair of scenarios. Depending on group allocation (feedback condition vs. no-feedback condition), the feedback device was either visible or hidden from the participant, but always recording. Accordingly, the four scenarios were:

(1) Participant performing chest compressions without feedback $(=\mathrm{C})$.

(2) Participant performing chest compressions with feedback (=CF).

(3) Participant performing ventilations without feedback $(=\mathrm{V})$.

(4) Participant performing ventilations with feedback (=VF).

Each scenario lasted for $3 \mathrm{~min}$ and all tasks were done in one session. Immediately after each scenario, participants were asked to report perceived workload using the standardized NASA-Task Load Index (NASA-TLX). ${ }^{26}$ We measured the mark made by participants on the scale for the NASA-TLX score.

\section{Equipment}

For CC scenarios we used the QCPR Baby manikin (Laerdal Medical, Stavanger, Norway), whereas for the ventilation scenarios we used the SimNewB manikin (Laerdal Medical, Stavanger, Norway) as it has no internal air leak. The QCPR Baby was connected to the SimPad Plus Skill Reporter device (Laerdal, Stavanger, Norway), which provided real-time visual feedback for CC performance. For ventilation feedback, a flow sensor (Neo Training, Monivent AB, Gothenburg, Sweden) was placed between the face mask (CareFusion Vital Signs Infant Face Mask, Chateaubriant, France) and the bag (Laerdal Silicone Resuscitator Pediatric Basic, Stavanger, Norway) to measure and show inspiratory $\left(V_{T_{\mathrm{i}}}\right)$ and expiratory tidal volume $\left(V_{T e}\right.$, graphically and numerically), peak inspiratory pressure (numerically), and mask leak (leak, numerically) in real time. A tablet (iPad, Apple Inc., Cupertino, CA) recorded the parameters from the sensor wirelessly for further analysis. Participants wore Tobii 2.0 mobile eyetracking glasses (Tobii, Danderyd, Sweden) to record real-time gaze behavior in all scenarios.

\section{Outcomes}

The primary outcome was the quality of CCs and ventilations. The quality of CCs was measured with a total compression score (\%), which is automatically calculated by the device including CC rate, CC rate compliance (percentage of correct CC rate), depth, depth compliance (percentage of correct CC depth), complete release, and hand position. Ventilation parameters included positive inspiratory pressure $\quad<30$ $\left.\mathrm{CmH}_{2} \mathrm{O}\right),{ }^{27}$ tidal volumes $(4-8 \mathrm{~mL} / \mathrm{kg}),{ }^{12,28}$ and leakage (as low as possible). We evaluated whether the use of feedback devices improved or worsened the quality of CPR as compared to no-feedback conditions.

As secondary outcomes, we evaluated participants' dwell time (amount of time looking at an area of interest [AOI]) and subjective workload. AOI describes a specific $\mathrm{AOI}$ in the video recording that is defined by researchers in the analysis process. For dwell time, the study group determined six AOls: (1) feedback device (if available), (2) ventilation bag, (3) infant chest, (4) ventilation mask, (5) study nurse, and (6) others. Participants' workload was measured with the NASA-TLX. ${ }^{26}$

\section{Sample size}

The sample size calculation with the $\mathrm{G}^{*}$ Power software for a paired $t$ test with two repeated measures (feedback, no-feedback) revealed that a sample size of 34 participants would provide sufficient power $(0.80)$ to detect an effect at the alpha level of 0.05 with medium effect size $\left(d_{z}=\right.$ $0.50)^{29}$

\section{Statistical analysis}

Descriptive statistics were used to describe the sample and analyze the acceptance of feedback devices. Paired $t$ tests were used to examine whether resuscitation quality, VA, and workload changed across the feedback conditions. Multiple regression analyses were conducted to test whether changes in resuscitation quality were predicted by participants' expertise, VA, and workload. For $V_{\mathrm{Te}}$ and $V_{\mathrm{Ti}}$ scores, $X^{2}$ tests were applied to compare the 
proportion of scores within the $4-8 \mathrm{~mL} / \mathrm{kg}$ range across the feedback conditions. All analyses were performed with SPSS 24.0 (IBM Corp., Armonk, NY). The level of significance was set at $P<0.05$ (two-tailed). Cohen's $d_{z}$ (for paired $t$ tests) were used as the measure of effect size, with $0.20,0.50$, and 0.80 indicating small, medium, and large effect, respectively. Parameters with a skewed data distribution were log transformed before analysis.

\section{RESULTS}

Data from 40 participants (25 females and 15 males) were collected. The recruitment period was from May 2019 to June 2020. Participants' characteristics are shown in Table 1.

\section{Resuscitation quality}

CC quality. Participants showed significant improvements in several CC parameters when provided with real-time feedback (Table 2). In particular, they had a $22 \%$ higher total CC score and a $25 \%$ higher CC rate compliance than in the no-feedback condition (both $P<0.001)$. Self-reported CC quality increased by $7 \%(P=$ $0.03)$. Mean CC depth increased by $0.9 \mathrm{~mm}$ with feedback $(P=$ 0.01 ), without having an effect on CC depth compliance. Years of clinical practice did not predict any changes in CC parameters, indicating that participants benefited from real-time feedback regardless of their experience.

Ventilation quality. Data of one participant were excluded because of technical issues with the flow sensor. Participants showed improvements in $V_{\mathrm{Ti}}, V_{\mathrm{Te}}$, and mask leak when provided with realtime feedback (Table 3 ). The mean $V_{\mathrm{Ti}}$ improved with feedback, increasing from $18 \%$ without feedback to $33 \%$ with feedback $\left(x^{2}=\right.$ 6.27, $P=0.012$ ). The mean $V_{\mathrm{Te}}$ was similar in both feedback conditions, but the proportion of $V_{\mathrm{Te}}$ in the $4-8 \mathrm{~mL} / \mathrm{kg}$ compliance range increased from $36 \%$ in the no-feedback condition to $67 \%$ in the feedback condition $\left(X^{2}=16.05, P<0.001\right)$. Mask leak was $7.5 \%$ lower with feedback $(P=0.009)$. Participants with less experience were lower than a $V_{\mathrm{Te}}$ of $4-8 \mathrm{~mL} / \mathrm{kg}$ limit more frequently than their more experienced counterparts $(\beta=0.45, P<0.001)$.

\section{Visual attention}

$V A$ in the CC task. In the no-feedback condition, infant chest was the most frequently attended $\mathrm{AOI}(73 \%)$, followed by ventilation mask (22\%). The addition of a feedback device had a powerful effect on participants' $V A$, with the feedback device being now the most frequently attended AOI (49\%), followed by infant chest (33\%) and ventilation mask (13\%). Paired comparisons revealed that participants significantly reduced their attention to the infant chest and the mask in the feedback condition compared with the no-feedback condition (Table 2). Years of clinical practice did not predict changes in VA except for the feedback device AOl; more experienced participants tended to dwell on the device longer than less experienced participants $(\beta=0.34, P=0.04)$. Furthermore, participants who dwelled longer on the device significantly reduced their mean $C C$ rate $(\beta=-0.29, P=0.01)$, thereby exceeding the 120 CCs per minute limit less frequently and thus improving their CC rate compliance (113.2 with feedback vs. 120.2 without feedback).

$V A$ in the ventilation task. Similar to the CC scenario, the infant's chest was the most frequently attended $\mathrm{AOI}(52 \%)$, and the ventilation mask the second (40\%) in the no-feedback condition. In the feedback condition, the feedback device was the most frequently attended AOI (40\%), whereas participants' attention to the infant's chest and the mask declined significantly (Table 3). Years of clinical practice did not predict changes in VA except for the mask AOI; more experienced participants tended to dwell on the ventilation mask shorter than less experienced participants when provided with real-time feedback $(\beta=-0.31, P=0.03)$. Dwell time on the feedback device predicted changes in the mean
Table 1. Characteristics of study participants $(N=40)$.

\begin{tabular}{|c|c|c|c|}
\hline Characteristic & $N(\%)$ & Mean (SD) & Range \\
\hline \multicolumn{4}{|l|}{ Sex } \\
\hline Female & $25(63)$ & & \\
\hline Male & $15(38)$ & & \\
\hline \multicolumn{4}{|l|}{ Age } \\
\hline$<30$ years & $19(48)$ & & \\
\hline $31-40$ years & $16(40)$ & & \\
\hline $41-50$ years & $4(10)$ & & \\
\hline$>51$ years & $1(3)$ & & \\
\hline \multicolumn{4}{|l|}{ Occupation, level of training } \\
\hline Neonatal nurse & $1(3)$ & & \\
\hline Medical student & $9(23)$ & & \\
\hline Neonatal fellow & $22(55)$ & & \\
\hline Neonatal consultant & $8(20)$ & & \\
\hline $\begin{array}{l}\text { Clinical experience in neonatology } \\
\text { (years) }\end{array}$ & & $4.26(6.5)$ & $0-26$ \\
\hline $\begin{array}{l}\text { Simulation-based resuscitation } \\
\text { training (times) }\end{array}$ & & $5.34(5.5)$ & $0-30$ \\
\hline \multicolumn{4}{|l|}{ Last CCs on actual patient } \\
\hline Never & $13(33)$ & & \\
\hline$<3$ months & $6(15)$ & & \\
\hline 3-5 months & $5(13)$ & & \\
\hline 6-11 months & $8(20)$ & & \\
\hline$>12$ months & $8(20)$ & & \\
\hline \multicolumn{4}{|l|}{ Last CCs on manikin } \\
\hline Never & $0(0)$ & & \\
\hline$<3$ months & $18(45)$ & & \\
\hline $3-5$ months & $4(10)$ & & \\
\hline 6-11 months & $8(20)$ & & \\
\hline$>12$ months & $10(25)$ & & \\
\hline \multicolumn{4}{|l|}{ Perceived BLS competence } \\
\hline Not sure & $3(8)$ & & \\
\hline Adequate & $25(64)$ & & \\
\hline Excellent & $11(28)$ & & \\
\hline \multicolumn{4}{|l|}{ Perceived ALS competence } \\
\hline Not sure & $19(48)$ & & \\
\hline Adequate & $16(40)$ & & \\
\hline Excellent & $5(13)$ & & \\
\hline \multicolumn{4}{|c|}{ Experience with any feedback device before the study } \\
\hline No & $26(65)$ & & \\
\hline Yes & $14(35)$ & & \\
\hline
\end{tabular}

The clinical experience in neonatology and simulation-based resuscitation training variables were measured as continuous variables.

CCs chest compressions, BLS basic life support, ALS advanced life support.

$V_{\mathrm{Te}}(\beta=0.23, P=0.03)$, with participants who dwelled longer on the device having higher mean $V_{\mathrm{Te}}(7.0 \mathrm{~mL} / \mathrm{kg})$ than participants who attended to the device for a shorter time $(6.6 \mathrm{~mL} / \mathrm{kg})$.

\section{Workload}

Workload in the CC task. The average NASA workload in the feedback condition was $37 \%$, which was $\sim 3.5 \%$ higher than in the no-feedback condition $(P=0.02)$. This was especially due to the increases in mental demands $(P=0.006)$, performance demands 
Table 2. Differences in outcome variables between the "feedback" and "no-feedback" condition in the chest compression task.

\begin{tabular}{|c|c|c|c|c|c|}
\hline \multirow[t]{2}{*}{ Variable } & \multirow[t]{2}{*}{ No feedback (mean \pm SD) } & \multirow[t]{2}{*}{ Feedback (mean \pm SD) } & \multirow[t]{2}{*}{ Difference } & \multicolumn{2}{|c|}{$T$ test $^{\mathrm{a}}$} \\
\hline & & & & $P$ & $d_{\mathbf{z}}$ \\
\hline \multicolumn{6}{|l|}{ Chest compression (CC) quality } \\
\hline Correct hand position (\%) & $82.48 \pm 29.9$ & $95.75 \pm 7.8$ & +13.27 & 0.057 & 0.31 \\
\hline CC depth $(\mathrm{mm})$ & $40.85 \pm 2.9$ & $41.73 \pm 1.6$ & +0.88 & 0.013 & 0.41 \\
\hline CC depth compliance (\%) & $85.90 \pm 27.6$ & $92.38 \pm 14.3$ & +6.48 & 0.772 & 0.05 \\
\hline CC rate compliance (\%) & $47.03 \pm 36.1$ & $72.23 \pm 28.1$ & +25.20 & 0.000 & 0.84 \\
\hline Full release (\%) & $82.23 \pm 30.2$ & $89.78 \pm 17.3$ & +7.55 & 0.751 & 0.05 \\
\hline Self-reported CC quality (\%) & $64.19 \pm 19.8$ & $71.35 \pm 13.1$ & +7.16 & 0.031 & 0.35 \\
\hline \multicolumn{6}{|l|}{ Visual attention (dwell time) } \\
\hline Feedback device (\%) & - & $48.72 \pm 23.8$ & - & - & - \\
\hline Study nurse (\%) & $0.33 \pm 0.6$ & $0.22 \pm 0.4$ & -0.11 & 0.264 & 0.18 \\
\hline Others (\%) & $4.02 \pm 6.4$ & $5.47 \pm 8.9$ & +1.45 & 0.065 & 0.31 \\
\hline \multicolumn{6}{|l|}{ NASA TLX workload } \\
\hline Average (\%) & $33.23 \pm 14.1$ & $36.82 \pm 12.9$ & +3.59 & 0.018 & 0.39 \\
\hline Mental demand (\%) & $23.70 \pm 19.7$ & $31.93 \pm 21.8$ & +8.23 & 0.006 & 0.46 \\
\hline Physical demand (\%) & $48.06 \pm 21.2$ & $46.79 \pm 22.2$ & -1.72 & 0.572 & 0.09 \\
\hline Temporal demand (\%) & $30.24 \pm 20.9$ & $28.09 \pm 18.7$ & -1.85 & 0.263 & 0.18 \\
\hline Performance demand (\%) & $34.08 \pm 17.4$ & $40.10 \pm 15.7$ & +6.02 & 0.047 & 0.32 \\
\hline Effort (\%) & $46.23 \pm 20.9$ & $47.85 \pm 21.5$ & +1.65 & 0.471 & 0.11 \\
\hline Frustration (\%) & $17.30 \pm 16.5$ & $26.09 \pm 17.6$ & +8.79 & 0.002 & 0.54 \\
\hline
\end{tabular}

Data are presented as mean \pm SD. Positive difference values indicate an increase (and negative values a decrease) in the respective parameter from the nofeedback to feedback condition.

${ }^{a}$ Exact significances ( $p$ values) and effect sizes (Cohen's $d_{z}$ values) for paired $t$ tests. Boldface $P$ values indicate significant changes in the outcome variables between the feedback conditions.

$(P=0.05)$, and frustration $(P=0.002)$ (Table 2). More experienced participants experienced higher average workload $(\beta=0.37, P=$ $0.001)$, higher mental demands $(\beta=0.39, P=0.003)$, and put more effort into the task $(\beta=0.26, P=0.02)$ than less experienced participants. The average workload in the feedback condition did not predict changes in participants' actual CC quality. There were only two effects of the NASA subscales on self-reported CC quality: higher performance demand and higher frustration were related to lower self-reported CC quality in the feedback condition $(\beta=-0.44$, $P=0.003$, and $\beta=-0.50, P=0.001$, respectively).

Workload in the ventilation task. The average NASA workload in the feedback condition was $36 \%$, which was $\sim 8 \%$ higher than in the no-feedback condition $(P<0.001)$. This was especially due to increases in mental demands $(P<0.001)$, performance demands $(P=0.002)$, and frustration $(P<0.001)$ (Table 3$)$. The increase in workload was unrelated to years of clinical practice. The average workload, performance demand, and frustration were related to a decline in self-reported ventilation quality $(\beta=-0.58, P<0.001$, $\beta=-0.63, P<0.001$, and $\beta=-0.63, P<0.001$, respectively). In addition, higher frustration was related to higher $V_{\mathrm{Ti}}(\beta=0.28, P=$ $0.04)$, with participants with $V_{T_{i}}$ in the $4-8 \mathrm{~mL} / \mathrm{kg}$ range being less frustrated than participants with $V_{\mathrm{Ti}}$ out of that range ( $28 \mathrm{vs} .53 \%$ ).

\section{The acceptance of feedback device}

The majority of participants found the use of both feedback devices, SimPad and Monivent, helpful for visualization of the CC and ventilation quality, respectively. More than $90 \%$ of participants perceived the feedback from the SimPad device as easily interpretable. The answers to the Monivent device were more ambiguous, with $62 \%$ of participants rating the feedback as rather easy to interpret and $36 \%$ of participants perceiving it rather difficult. Participants further stated that using feedback devices would also be beneficial in an actual scenario in a pediatric patient (Table 4).

\section{DISCUSSION}

In this study, we observed the effect of feedback devices on the quality of pediatric resuscitation with eye tracking to analyze participants' attention and workload. There were three main findings: (i) As in previous studies, we found that performance quality improved with real-time feedback for both CCs and ventilations. Feedback devices are designed to assist in delivering optimal resuscitation quality. ${ }^{6-10,12-15}$ A recently published systematic review also supported the use of feedback devices for pediatric as well as adult resuscitation support. ${ }^{30}$ The feedback devices helped the practitioners to appropriately recognize and optimize resuscitative tasks rather than serving as a distraction.

(ii) The addition of a feedback device influenced VA. When feedback devices were used, participants' attention shifted significantly to the device (see Fig. 2) and away from the manikin consistent with former studies. ${ }^{31,32}$ However, this shift in attention was not harmful to performance in our study. We further observed 
Table 3. Differences in outcome variables between the "feedback" and "no-feedback" condition in the ventilation task.

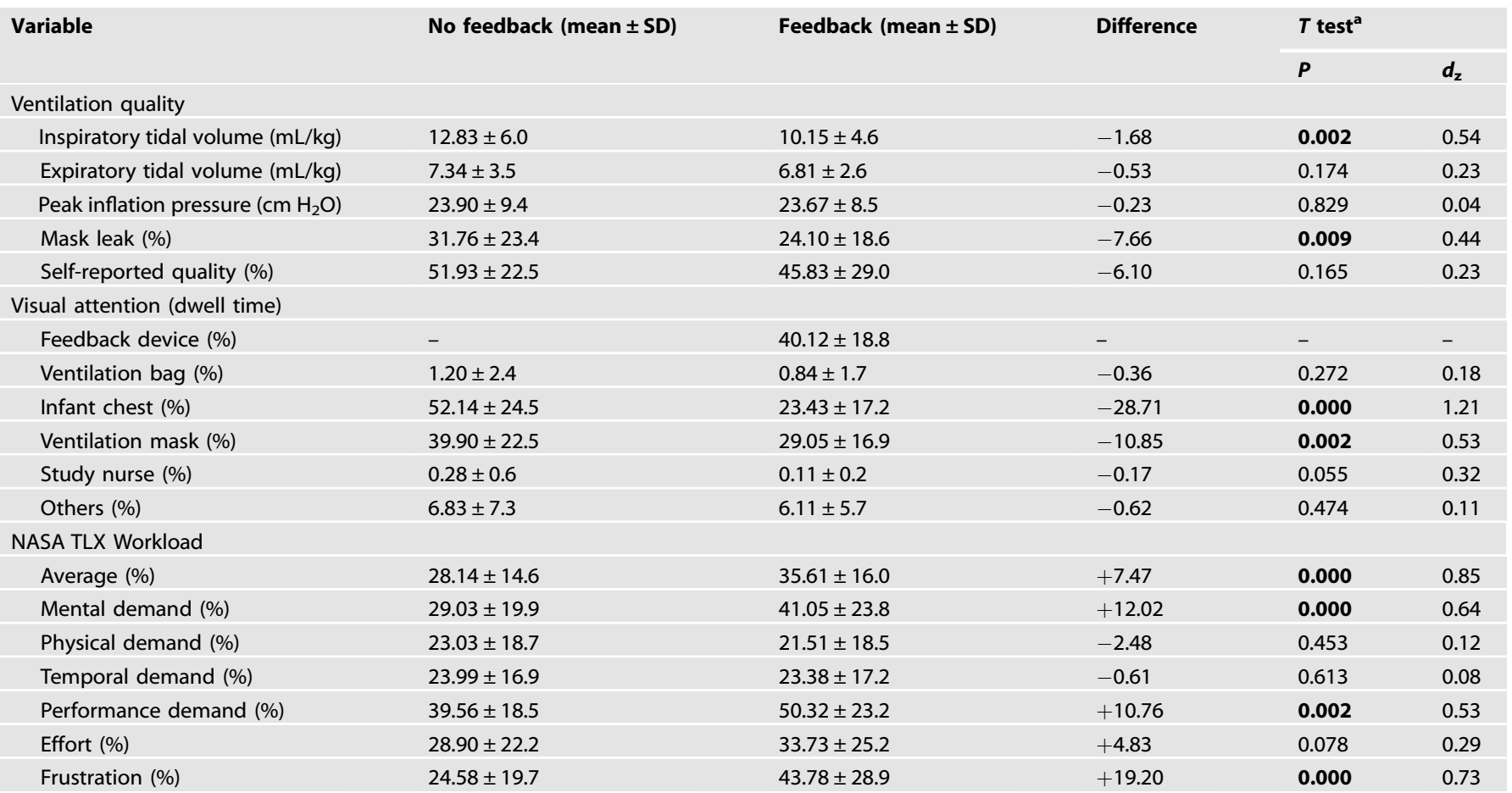

Data are presented as mean \pm SD. Positive difference values indicate an increase (and negative values a decrease) in the respective parameter from the nofeedback to feedback condition

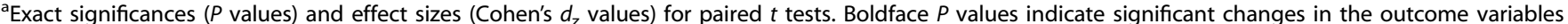
between the feedback conditions.

Table 4. Participants' subjective experience with the SimPad (chest compressions) and Monivent Neo (ventilation) feedback device.

\section{SimPad, $\boldsymbol{N}(\%) \quad$ Monivent Neo, $\mathbf{N}(\%)$}

Did you find the feedback device helpful in performing chest compressions/ventilations?

$\begin{array}{lll}\text { Very helpful } & 20(50) & 21(54) \\ \text { Somewhat helpful } & 15(38) & 12(31) \\ \text { Moderate } & 2(5) & 1(3) \\ \text { Slightly helpful } & 2(5) & 4(10) \\ \text { Not at all helpful } & 1(3) & 1(3)\end{array}$

How difficult or easy was it to interpret feedback from the device?

$\begin{array}{lll}\text { Very difficult } & 0(0) & 0(0) \\ \text { Difficult } & 0(0) & 14(36) \\ \text { Moderate } & 3(8) & 1(3) \\ \text { Easy } & 22(55) & 15(39) \\ \text { Very easy } & 15(38) & 9(23)\end{array}$

Do you think using a feedback device for chest compressions/ ventilations would be beneficial in a real scenario?
Yes
$35(88)$
$28(72)$
No
$4(10)$
Don't know
3 (8)
7 (18)

Did you get distracted by the feedback devices?
Very distracted
5 (13)
Somewhat distracted
$21(53)$
Moderate
5 (13)
Slightly distracted
$6(15)$
Not at all distracted
$3(8)$

that more experienced participants spent more time looking at the feedback device than on the manikin. These participants may have needed more time to interpret the feedback from the device or relied on their haptic memory requiring less confirmation of their task by looking directly at the manikin. However, also the non-feedback group's dwell time was mostly on the infant's chest and mask, likely to determine adequate chest rise. Probably, the participants were looking for feedback or needed feedback on how they were doing by paying attention to the areas that would tell them the quality of ventilation. While it sounds reasonable that participants are actively seeking feedback on performance, there is no literature that confirms this so far.

(iii) The addition of a feedback device increased subjective workload, but this increase was small ${ }^{33}$ and did not interfere with resuscitation quality. The increase was markedly higher when the participant had more years of practice, but only when performing CCs. Interestingly, a higher subjective workload was commonly paired with frustration when using feedback devices, but the quality of CCs and ventilation did not suffer in our study. The relationship between workload and clinical performance has been reported controversially in the literature. Previous studies have described a potential reduction of workload when using feedback devices, ${ }^{34}$ but also an increased workload with improved quality of CC. $^{20}$ While feedback devices have beneficial effects on resuscitation performance, the effect might decrease once feedback devices are used on a daily basis and are not special to providers anymore. ${ }^{35}$ Ultimately, these devices make CPR performance measurably and their clinical use may allow the identification of the best practice during resuscitation. ${ }^{11}$

Overall, our findings support previous reports that feedback devices may be an important tool in CPR in situ as well as in real life, $^{36}$ also with an improvement of return of spontaneous circulation rates. $^{37}$ 


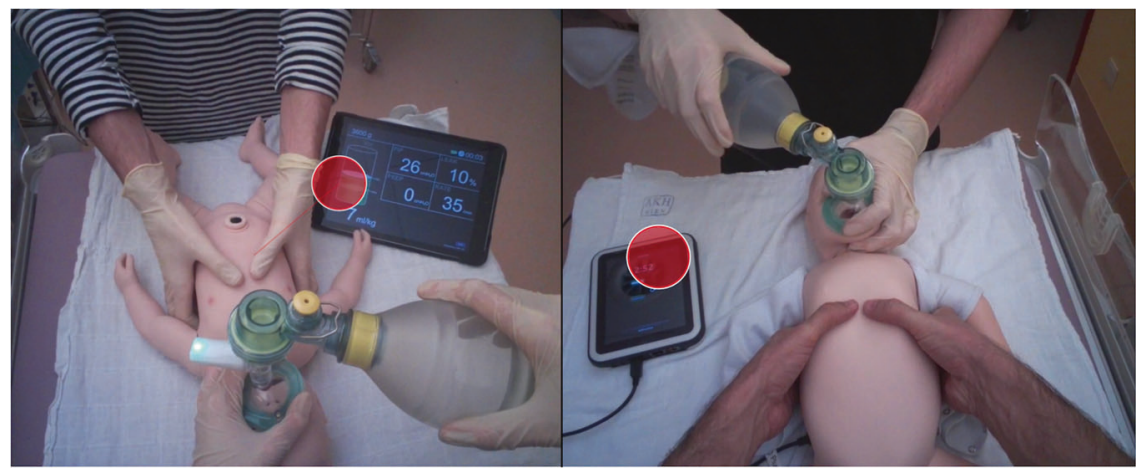

Fig. 2 Snapshots showing visual attention (red circle) of participants when feedback devices were used. Participants' attention shifted significantly to the device and away from the manikin. Dwell time (=total duration of visit time) on the feedback device was $40.1 \%$ in the ventilation feedback condition (left) and $48.7 \%$ in the chest compression feedback condition (right). In both conditions, participants significantly reduced attention from the infant's chest and mask ( 72.9 vs. $32.6 \%$ and 21.9 vs. $12.7 \%)$.

However, while we are not able to provide clinical data, it was interesting to see that while feedback devices increased subjective workload, resuscitation quality was mostly not influenced. Future studies should also focus on interviews with participants after the scenario, to identify why and what exactly influenced workload. Most providers were using feedback devices for the first time, which might in part explain the higher perceived workload. It is also reasonable that repetitive and regular training with feedback devices might decrease subjective workload while still impacting the quality of resuscitation positively. Alternatively, this increase in workload with feedback devices may be offset by a resuscitation coach who relays CPR quality information to the resuscitating provider. ${ }^{38,39}$ We assume that adding live feedback devices in real clinical situations, such as cardiopulmonary resuscitation and positive pressure ventilation, can help to increase the quality of patient care. However, clinical studies analyzing the utility of feedback devices in such situations including VA and workload are needed.

\section{Limitations}

This was a simulation-based trial not involving any real patients. Furthermore, the study design did not allow for blinding. Participants knew they were being studied, which might amplify the shift of VA to the feedback device. In addition, we were not able to evaluate any other feedback routes such as voice or sound cues. Furthermore, scenarios were limited to $3 \mathrm{~min}$, so we were unable to identify possible changes of workload with a longer duration of resuscitation. Workload measurement was based on subjective reports by participants.

\section{CONCLUSION}

We found that resuscitation quality significantly improved when using feedback devices in a simulated setting. VA shifted to the feedback device, when provided, and away from the simulated patient and other equipment. The subjective workload was higher with the feedback device. High-quality randomized controlled trials, as well as real-patient trials focusing on patient outcomes, are needed to determine the impact of VA and stressors when using feedback devices.

\section{REFERENCES}

1. Slonim, A. D., Patel, K. M., Ruttimann, U. E. \& Pollack, M. M. Cardiopulmonary resuscitation in pediatric intensive care units. Crit. Care Med. 25, 1951-1955 (1997).

2. Peddy, S. B. et al. Cardiopulmonary resuscitation: special considerations for infants and children with cardiac disease. Cardiol. Young 17(Suppl. 2), 116-126 (2007).
3. Gupta, P. et al. Outcomes following single and recurrent in-hospital cardiac arrests in children with heart disease: a report from American Heart Association's get with the guidelines registry-resuscitation. Pediatr. Crit. Care Med. 17, 531-539 (2016).

4. Young, A. K. et al. Use of a simulation-based advanced resuscitation training curriculum: Impact on cardiopulmonary resuscitation quality and patient outcomes. J. Intensive Care Soc. 21, 57-63 (2020).

5. Moretti, M. A. et al. Advanced cardiac life support training improves long-term survival from in-hospital cardiac arrest. Resuscitation 72, 458-465 (2007).

6. Wagner, M. et al. Effects of feedback on chest compression quality: a randomized simulation study. Pediatrics 143, e20182441 (2019).

7. Goharani, R. et al. Real-time compression feedback for patients with in-hospital cardiac arrest: a multi-center randomized controlled clinical trial. J. Intensive Care 7, 5 (2019).

8. Cheng, A. et al. Improving cardiopulmonary resuscitation with a CPR feedback device and refresher simulations (CPR CARES Study): a Randomized Clinical Trial. JAMA Pediatr. 169, 137-144 (2015).

9. Bobrow, B. J. et al. The influence of scenario-based training and real-time audiovisual feedback on out-of-hospital cardiopulmonary resuscitation quality and survival from out-of-hospital cardiac arrest. Ann. Emerg. Med. 62, 47-56.e41 (2013).

10. Allan, K. S., Wong, N., Aves, T. \& Dorian, P. The benefits of a simplified method for CPR training of medical professionals: a randomized controlled study. Resuscitation 84, 1119-1124 (2013).

11. Lin, S. \& Scales, D. C. Cardiopulmonary resuscitation quality and beyond: the need to improve real-time feedback and physiologic monitoring. Crit. Care 20, 182 (2016).

12. Schmölzer, G. M. et al. Respiratory function monitor guidance of mask ventilation in the delivery room: a feasibility study. J. Pediatr. 160, 377-381.e372 (2012).

13. Khoury, A., De Luca, A., Sall, F. S., Pazart, L. \& Capellier, G. Ventilation feedback device for manual ventilation in simulated respiratory arrest: a crossover manikin study. Scand. J. Trauma Resusc. Emerg. Med. 27, 93 (2019).

14. Gould, J. R., Campana, L., Rabickow, D., Raymond, R. \& Partridge, R. Manual ventilation quality is improved with a real-time visual feedback system during simulated resuscitation. Int. J. Emerg. Med. 13, 18 (2020).

15. Bowman, T. A., Paget-Brown, A., Carroll, J., Gurka, M. J. \& Kattwinkel, J. Sensing and responding to compliance changes during manual ventilation using a lung model: can we teach healthcare providers to improve? J. Pediatr. 160, 372-376. e371 (2012).

16. Perkins, G. D. et al. European Resuscitation Council Guidelines for Resuscitation: 2017 update. Resuscitation 123, 43-50 (2018).

17. Van de Voorde, P. et al. European Resuscitation Council Guidelines 2021: paediatric life support. Resuscitation 161, 327-387 (2021).

18. Lin, Y., Cheng, A., Grant, V. J., Currie, G. R. \& Hecker, K. G. Improving CPR quality with distributed practice and real-time feedback in pediatric healthcare providers - a randomized controlled trial. Resuscitation 130, 6-12 (2018).

19. Gurung, R. et al. Effectiveness and acceptability of bag-and-mask ventilation with visual monitor for improving neonatal resuscitation in simulated setting in six hospitals of Nepal. J. Nepal Health Res. Counc. 17, 222-227 (2019).

20. Brown, L. L. et al. Impact of a CPR feedback device on healthcare provider workload during simulated cardiac arrest. Resuscitation 130, 111-117 (2018).

21. Byrne, A. J. et al. Novel method of measuring the mental workload of anaesthetists during clinical practice. Br. J. Anaesth. 105, 767-771 (2010). 
22. Wagner, M. et al. Eye-tracking during simulation-based neonatal airway management. Pediatr. Res. 87, 518-522 (2020).

23. Law, B. H. Y. et al. Analysis of neonatal resuscitation using eye tracking: a pilot study. Arch. Dis. Child Fetal Neonatal Ed. 103, F82-f84 (2018).

24. Cheng, A. et al. Reporting Guidelines for Health Care Simulation Research: extensions to the CONSORT and STROBE statements. Simul. Health. 11, 238-248 (2016).

25. Maconochie, I. K. et al. European Resuscitation Council Guidelines for Resuscitation 2015: Section 6. Paediatric life support. Resuscitation 95, 223-248 (2015).

26. Hart, S. G. et al. Development of NASA TLX. Adv. Psychol. 52, 139-183 (1988)

27. Wyllie, J. et al. European Resuscitation Council Guidelines for Resuscitation 2015: Section 7. Resuscitation and support of transition of babies at birth. Resuscitation 95, 249-263 (2015).

28. Chowdhury, O. et al. Volume-targeted ventilation in infants born at or near term. Arch. Dis. Child. Fetal Neonatal Ed. 97, F264 (2012).

29. Ferguson, C. J. An effect size primer: a guide for clinicians and researchers. Prof. Psychol. Res. Pract. 40, 532-538 (2009).

30. Gugelmin-Almeida, D., Tobase, L., Polastri, T. F., Peres, H. H. C. \& Timerman, S. Do automated real-time feedback devices improve CPR quality? A systematic review of literature. Resuscitation 6, 100108 (2021).

31. Herrick, $H$. et al. Provider visual attention on a respiratory function monitor during neonatal resuscitation. Arch. Dis. Child Fetal Neonatal Ed. 105, 666-668 (2020).

32. Katz, T. A. et al. Visual attention on a respiratory function monitor during simulated neonatal resuscitation: an eye-tracking study. Arch. Dis. Child Fetal Neonatal Ed. 104, F259-F264 (2019).

33. Mazur, L. M., Mosaly, P. R., Hoyle, L. M., Jones, E. L. \& Marks, L. B. Subjective and objective quantification of physician's workload and performance during radiation therapy planning tasks. Pract. Radiat. Oncol. 3, e171-e177 (2013).

34. Yamada, N. K., Fuerch, J. H. \& Halamek, L. P. Ergonomic challenges inherent in neonatal resuscitation. Children 6, 74 (2019).

35. Troy, L. et al. Healthcare provider perceptions of cardiopulmonary resuscitation quality during simulation training. Pediatr. Crit. Care Med. 20, e473-e479 (2019).

36. Hostler, D. et al. Effect of real-time feedback during cardiopulmonary resuscitation outside hospital: prospective, cluster-randomised trial. BMJ 342, d512 (2011).

37. Vahedian-Azimi, A. et al. Effect of the Cardio First Angel device on CPR indices: a randomized controlled clinical trial. Crit. Care 20, 147 (2016).

38. Tofil, N. M. et al. Effect of a cardiopulmonary resuscitation coach on workload during pediatric cardiopulmonary arrest: a multicenter, simulation-based study. Pediatr. Crit. Care Med. 21, e274-e281 (2020).

39. Hunt, E. A. et al. Improved Cardiopulmonary Resuscitation Performance With CODE ACES(2): a resuscitation quality bundle. J. Am. Heart Assoc. 7, e009860 (2018).

\section{ACKNOWLEDGEMENTS}

We acknowledge support from Monivent AB (Gothenburg, Sweden), who provided us with training as well as technical support during the study. We further thank Lukas Zwingl and the entire pediatric simulation team for their continuous help during the study. This work was supported by ZOLL Foundation, grant number 74774258.2.
The funder did not have any influence in study design, data collection, analysis, decision to publish, or preparation of the manuscript.

\section{AUTHOR CONTRIBUTIONS}

M.W., F.E., F.S.C., and P.G. conceptualized and designed the study, drafted the initial manuscript, collected, and analyzed the data, and reviewed and revised the manuscript. L.K. collected data, carried out eye-tracking analyses, and reviewed and revised the manuscript. I.T.G., K.B., and A.B. helped with analyzing the data and coordination of the study, and critically reviewed and revised the manuscript for important intellectual content. All authors approved the final manuscript as submitted and agreed to be accountable for all aspects of the work.

\section{COMPETING INTERESTS}

The authors declare no competing interests.

\section{CONSENT STATEMENT}

The ethics committee of the Medical University of Vienna gave this study an exempt status. As this study was a simulation-based study and no trial registration was done.

\section{ADDITIONAL INFORMATION}

Supplementary information The online version contains supplementary material available at https://doi.org/10.1038/s41390-021-01653-w.

Correspondence and requests for materials should be addressed to M.W.

Reprints and permission information is available at http://www.nature.com/ reprints

Publisher's note Springer Nature remains neutral with regard to jurisdictional claims in published maps and institutional affiliations.

(i) Open Access This article is licensed under a Creative Commons Attribution 4.0 International License, which permits use, sharing, adaptation, distribution and reproduction in any medium or format, as long as you give appropriate credit to the original author(s) and the source, provide a link to the Creative Commons license, and indicate if changes were made. The images or other third party material in this article are included in the article's Creative Commons license, unless indicated otherwise in a credit line to the material. If material is not included in the article's Creative Commons license and your intended use is not permitted by statutory regulation or exceeds the permitted use, you will need to obtain permission directly from the copyright holder. To view a copy of this license, visit http://creativecommons. org/licenses/by/4.0/.

(c) The Author(s) 2021 